fibers compared to the nanoparticle films. As a result, transport of photoinjected electrons to the back contact was faster and recombination with the electrolyte slowed down significantly. To further improve device efficiency, the researchers are interested in decreasing the diameter of the nanofibers to increase the roughness factor of the films. To enhance the durability of the DSSC devices, the group also intends to employ ionic liquid electrolytes to obtain long-term stability at full sunlight intensity.

MELISSA A. HARRISON

\section{Functionalized Silsesquioxanes Show High Sensitivity and Stability for Next-Generation E-Beam Lithography Resists}

One of the most prevalent methods for creating submicron patterns is e-beam lithography (EBL). Electron optics can produce electron beams with a diameter of a few nanometers. The spatial resolution of EBL (typically $\approx 10 \mathrm{~s} \mathrm{~nm}$ ) is limited by scattering in the photoresist. Thus, it remains a challenge to create EBL resists which maintain high contrast below $20 \mathrm{~nm}$. Hydrogen silsesquioxane (HSQ) has shown promise as a negative resist due to its ability to create sub-20 nm features with high contrast and fidelity. However, HSQ has low sensitivity and is not stable for long periods of time.

J.H. Sim of Seoul National University, H.-J. Lee of the National Institute of Standards and Technology, and their colleagues propose a solution to these problems in the May 25 issue of Chemistry of Materials (DOI: $10.1021 / \mathrm{cm} 9035456$; p. 3021). Sim and coresearchers created two compositions of functionalized HSQ resists (shown in Scheme 1). Norbornene-modified HSQ is created by co-polymerizing triethoxysilane (TES) with norbornene ethyltrimethoxysilane (NETMS). Norbornene-co-chloromethylphenyl functionalized silsesquioxane is obtained by co-polymerizing $p$-chloromethylphenyl trimethoxysilane and norbornene ethyltrimethoxsilane.

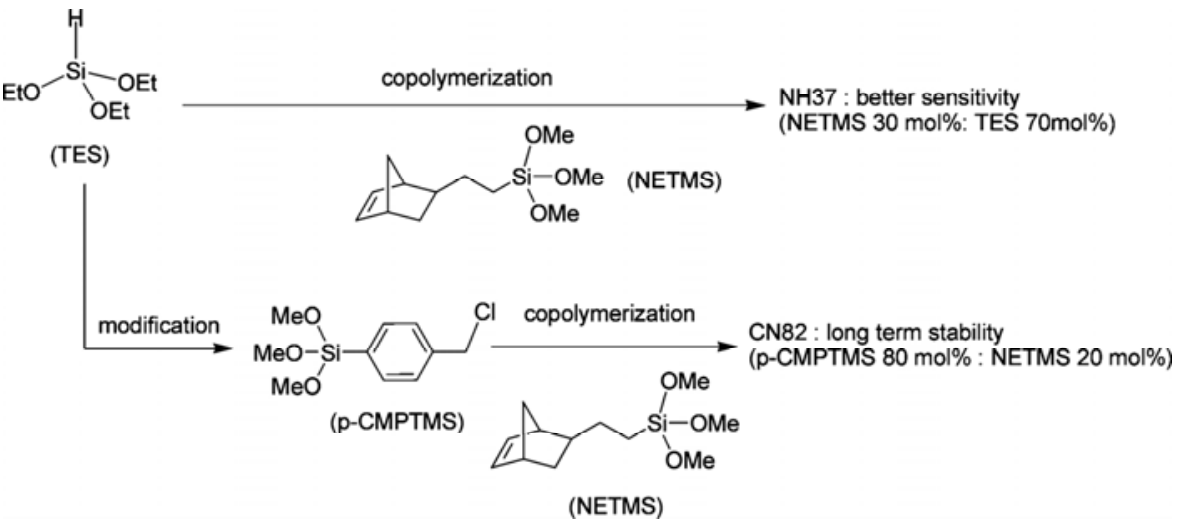

Scheme 1. Schematic showing compounds used for creating norbornene-functionalized HSQ (top line) and norbornene-co-chloromethylphenyl functionalized silsesquioxane (bottom line). Reproduced with permission from Chemistry of Materials 22 (10) (2010) 3021; DOI:10.1021/cm9035456. (C) 2010 American Chemical Society.

Contrast curves demonstrate that the norbornene and norbornene-co-chloromethylphenyl resists become insoluble at $58 \%$ and $75 \%$ of the electron dose for standard HSQ, respectively. Scanning electron micrographs qualitatively show that these resists also maintained high-resolution patterning at low electron dosages. The norbornene-functionalized resists produced $15 \mathrm{~nm}$ lines and norbornene-co-chloromethylphenyl resists produced $20 \mathrm{~nm}$ wide lines. While functionalizing HSQ with norbornene increased the shelf life in solution from approximately five days to 10 days, functionalizing with norbornene-co-chloromethylphenyl allowed the molecule to be stable for over a year without forming a gel.

The researchers conclude that by functionalizing the HSQ molecule, resists can be created with higher sensitivity and stability than currently possible, without sacrificing nanometer-scale resolution. According to the researchers, the ability to create more sensitive resists opens the door to creating large-area patterns using EBL.

SCOTT COOPER

\section{Method Developed for Producing Chitosan Nanoparticles Using Nanoporous Membranes}

The drive to develop drug delivery systems that can be tailored for controlled release of pharmaceutical action continues to receive immense research, but the efficacy of many drugs is limited by their ability to reach the site of optimum therapeutic action. Recently, R.N. Zare of Stanford University and C.R. Martin of the University of Florida and their colleagues have developed a liquid-liquid separation approach using a nanoporous membrane to produce organic nanoparticles, which can be loaded with other organic guest molecules. To illustrate this technique, the researchers used chitosan, a $\mathrm{pH}$-responsive polymer with biodegradable and biocompatible properties. These properties enable chitosan to be used in a wide range of applications in both pharmaceutical and biomedical fields.

As reported in the May 4 online edition of Nano Letters (DOI: 10.1021/nl101057d), the research team used track-etched polycarbonate (PCTE) and anodized aluminum oxide (AAO) nanoporous membranes having $10 \mathrm{~nm}$ and $20 \mathrm{~nm}$ cylindrical pores, respectively, as the separator or the liquid liquid system. The $\mathrm{pH}$ of the feed and receiver solutions was adjusted independently so that chitosan is soluble in the feed solution and insoluble in the receiver solution. The feed solution was forced under pressure through the pores of the membrane into the receiver solution, as shown in Figure 1. When nanodroplets of the soluble chitosan were injected through the membrane into the receiver solution, nanoparticles of chitosan were formed at the exits of the nanopores and carried 